\title{
L'absence de preuve est-elle la preuve de l'absence ?
}

\author{
Does the absence of evidence for its presence prove it is not there?
}

(C) Springer-Verlag France 2010

\section{Surcoût de l'endoscopie réalisée en ambulatoire, consécutive à une consultation aux urgences hospitalières ou à une hospitalisation secondaire dans les 14 jours suivant l'endoscopie \\ Leffler DA, Kheraj R, Garud S, Neeman N, Nathanson LA, Kelly CP, et al. Arch Intern Med 2010 170:1752-7}

Alors qu'en matière d'endoscopie l'ASGE a récemment défini ce que l'on pouvait classer en effet secondaire mineur, majeur et complication, la fréquence de celles-ci dépend de la période d'enregistrement. Il était déjà connu qu'un nombre négligeable de complications générales cardiorespiratoires pouvait survenir après la sortie du patient. Bien qu'exceptionnel, ce taux de complications doit être pris en compte, car le développement de l'hospitalisation ambulatoire est croissant, la population cible de ces traitements est de plus en plus âgée et atteints de comorbidité cardiorespiratoire, et la minimisation des coûts devrait les prendre en compte. L'article de Leffler et al. a permis d'approcher cette dépense en enregistrant pendant neuf mois la fréquence des réhospitalisations après endoscopie œsogastroduodénale (FOGD) ou vidéocoloscopie totale (CT). Parmi 6383 FOGD et 11632 CT (dont 7392 CT de dépistage) réalisées en ambulatoire, les visites ou consultations aux urgences de l'hôpital dans les 14 jours suivants ont été enregistrées automatiquement. Parmi 419 visites, 134 (32\%) ont été considérées en relation avec l'endoscopie précédente : 68 visites après 6383 FOGD et 98 visites après $11632 \mathrm{CT}$ (dont 70 après 7392 pour CT dépistage). Alors que 32 sur 134 patients avaient eu une FOGD et une CT concomitantes, elles ont été comptabilisées une fois comme visite après FOGD et une fois après $\mathrm{CT}$, totalisant 166 visites pour effet secondaire dans les 14 jours suivants. Parallèlement, les visites ou consultations au service des urgences de l'hôpital dans les 14 jours étaient enregistrées après déclaration spontanée par les gastroentérologues ayant réalisées les FOGD ou CT : 31 des 134 visites et huit autres ont consulté dans un autre établissement et ont été déclarées par ces gastroentérologues. Le délai moyen était de 6,0 jours (médiane : 5,5) pour les FOGD et de 5,2 jours (médiane : 3,5 ) pour les CT. Les causes de consultations étaient des douleurs abdominales $(n=63$, soit $47 \%$ des visites), 16 hémorragies digestives ( $12 \%$ ), dont 12 et quatre parmi 6383 FOGD et $11632 \mathrm{CT}$ ambulatoires, des douleurs thoraciques ( $n=14$ ou $11 \%$ ), huit fois une hyperthermie (6\%), sept pneumonies (5\%), quatre perforations (3\%) et une crise d'asthme, un infarctus du myocarde, une phlébite, une rupture d'hépatome, une pancréatite aiguë et un décès à j8 après arrêt d'aspirine. Le surcoût moyen était de 6356 dollars pour 134 consultations sans hospitalisation (extrêmes : 196-174 213), soit une médiane de 5270 dollars pour 134 visites (IC $95 \%: 4$ 067-8 644). Cela augmentait le coût de 48 dollars (IC $95 \%$ : 34-62) par CT, soit un surcoût de 4,2 \% (remboursement par le Medicare de 1140 dollars) et de 79 dollars par FOGD.

Au total, 142 patients avec effet secondaire ont nécessité une consultation ou passage à l'hôpital dont seulement $39(31+8)$, soit $27 \%$ par déclarations par les gastroentérologues. Cela représentait un risque ou une probabilité de 0,79\% (IC $95 \%: 0,63-0,88$ ) pour les 18015 endoscopies (6 383 FOGD et $11632 \mathrm{CT}$ ) réalisées en mode ambulatoire, soit 1,07\% (IC $95 \%: 0,84-1,35$ ) pour les 6383 FOGD et $0,84 \%$ (IC $95 \%: 0,69-1,03$ ) pour les $11632 \mathrm{CT}$ ambulatoires, ou $0,95 \%$ (IC $95 \%: 0,75-1,19$ ) pour les $7392 \mathrm{CT}$ de dépistage. La cause était majoritairement bénigne avec un effet secondaire grave dans 32 cas $(0,17 \%)$ et un taux de mortalité quasi nul (1/18 000).

Le taux non pas de consultation ou d'hospitalisation dans les 14 jours suivant un acte endoscopique mais celui de déclaration par le gastroentérologue ayant effectué l'acte pourrait également constituer un critère de qualité, encore faudrait-il s'en donner les moyens ou bénéficier d'une politique intelligente et efficace. En effet, à l'échelle française, pour 1,2 million de $\mathrm{CT}$, un surcoût de $4,2 \%$ représente une enveloppe moyenne correspondant à 50400 coloscopies, avec selon les comorbidités moyennes, un surcoût de 25,2 millions d'euros, alors que la rémunération du rappel systématique à j6 ou j7 par un auxiliaire médical s'élèverait à... c'est simple comme un coup de fil! 